\title{
Echinococcus multilocularis: immunological study on the "Em2-positive" laminated layer during in vitro and in vivo post-oncospheral and larval development *
}

\author{
B. Gottstein ${ }^{1}$, P. Deplazes ${ }^{1}$, and M. Aubert ${ }^{2}$ \\ 1 Institute of Parasitology, University of Zürich, Zürich, Switzerland \\ ${ }^{2}$ Centre National d'Etudes Vétérinaires et Alimentaires, Malzeville, France
}

Accepted January 15, 1992

\begin{abstract}
Echinococcus multilocularis oncospheres, primary vesicular cysts, and protoscolices were assessed in vitro and in vivo for their potential to synthesize a PASpositive laminated layer containing monoclonal antibody $(\mathrm{mAb}) \mathrm{G} 11$-binding Em2 antigen. The presence of Em2 antigen in developed oncospheres and cysts was subsequently correlated to the potential of in vivo development into a secondary metacestode in recipient host mice, which also responded by anti-Em 2 serum antibody formation. In contrast, protoscolices failed to develop the "Em2-positive" layer in vitro under the selected experimental conditions. The failure to develop subsequently in vivo into a secondary metacestode was underlined by a lack of anti-Em2 serum antibody formation by the hosts. We furthermore developed a technique to obtain E. multilocularis clones by inoculating single oncospheres into recipient mice.
\end{abstract}

Relatively few reports of in vitro culture of Echinococcus multilocularis metacestodes have addressed the in vivo host-parasite interplay. We have recently demonstrated that in vitro post-oncospheral development is accompanied by the formation of a laminated layer within 13 days of culture (Deplazes and Gottstein 1991). This laminated layer contains a dominant antigenic substance called Em2 antigen (Gottstein 1985), which can be identified and demonstrated immunologically using monoclonal antibody mAb G11 (Deplazes and Gottstein 1990, 1991). The laminated layer may play an important role in protecting the developing oncosphere from host immune reactions (Sakamoto and Sugimura 1970). Yamashita et al. (1962) have found that protoscolices exhibit a dual in vitro development potential in that they may differentiate into adults as well as into a cystic (metacestode) stage. In the latter case, the protoscolex

* This work was supported by grant 31-29651.90 from the Swiss National Science Foundation

Offprint requests to: $\mathrm{B}$. Gottstein either becomes vesicular or develops a posterior bladder that subsequently differentiates into a spherical cystic form. An alternative method of inducing metacestode proliferation in vitro uses germinal layer or vesiculated cyst tissue as initial parasite material (Rausch and Jentoft 1957; Ali-Khan and Siboo 1982; Kroeze and Tanner 1986).

The present paper describes some characteristics of initial larval structures (oncosphere, primary vesicular cyst, protosolices) following in vitro cultivation or in vivo proliferation with respect to the potential of forming a laminated layer containing $\mathrm{mAb}$ G11-binding Em2 antigen and its subsequent consequences.

\section{Materials and methods}

\section{Experimental design}

Echinococcus multilocularis oncospheres, purified protoscolices, and primary vesicular cysts were cultivated in vitro for up to 2 months. Parasite material, either freshly prepared or preliminarily cultivated in vitro, was sequentially tested for the presence of a synthesized "Em2-positive" laminated layer by light microscopy of native or PAS-stained material and direct immunofluorescence using fluorescein isothiocyanate (FITC)-labeled monoclonal antibody (mAb) G11. Em2 antigen synthesis was also sequentially monitored by testing culture medium supernatants with a doubleantibody sandwich-enzyme-linked immunosorbent assay (ELISA) using solid-phase and alkaline phosphatase-labeled mAb G11. In vitro cultured (for 14 days or more) as well as freshly prepared parasite material was injected intraperitoneally into susceptible mice to determine its ability to induce secondary alveolar echinococcosis. This was assessed by macroscopic examination of necropsied animals for the presence of metacestode tissue and by serological examination of the mice using $E$. multilocularis antigens reflecting different degrees of specificity for the larval stage of parasite development.

\section{E. multilocularis eggs/oncospheres}

E. multilocularis eggs were isolated under biohazard conditions from adult tapeworms recovered from the small intestine of natu- 
rally infected necropsied foxes. Purification and hatching of eggs and activation of oncospheres for in vitro cultivation was done as described elsewhere (Deplazes and Gottstein 1991).

\section{E. multilocularis protoscolices}

The FAub-2 (originating from the same endemic area as the eggs described above) and SLI-144 (obtained from a naturally infected Microtus oeconomus on St. Lawrence Island, Alaska, USA) isolates of E. multilocularis were grown in Meriones unguiculatus for 3 months so as to obtain a dense protoscolex formation. Further processing of the metacestode tissue for high purification of protoscolices was performed exactly according to a previously established technique (Thompson et al. 1990). To obtain a highly pure protoscolex suspension, we checked the whole suspension microscopically and removed any remaining particle with a sterile glass capillary. The viability and maturity of protoscolices were assessed by microscopical demonstration of fully developed structures and flame cell activity. The protoscolices most of which evaginated following treatment (according to Rodriguez-Caabeiro and Casado 1988), were assessed by light microscopy for maturity using the criteria of Marchiondo and Andersen (1984). The same investigations were performed using another E. multilocularis isolate, SLI144 , for confirmation of results.

\section{E. multilocularis primary acephalic vesicular cysts}

The FAub-2 isolate of $E$. multilocularis described above was grown in $\mathrm{C} 57 \mathrm{BL} / 6 \mathrm{~J}$ mice for 2 months in order to obtain highly vesiculated metacestode tissue without protoscolex formation (Ali-Khan and Siboo 1982). High purification of primary vesicular cysts consisted of mincing the tissue with a scalpel blade and subsequently pressing the residues through a sterile stainless-steel sieve (mesh size, $0.5 \mathrm{~mm}$ ). A large portion of calcareous corpuscules was removed by several rapid sedimentations of parasite material in Dulbecco's modified Eagle's medium (DMEM, Flow) at $1 \mathrm{~g}$.

\section{Culture conditions}

Activated oncospheres were cultivated for 14 days in $30-\mathrm{ml}$ plastic tissue culture flasks (Falcon) containing a monolayer of Swiss mouse embryo (3T3) cells (Flow) in $10 \mathrm{ml}$ tissue-culture medium [DMEM supplemented with $100 \mathrm{IU}$ penicillin $\mathrm{G} / \mathrm{ml}, 100 \mu \mathrm{g}$ streptomycin/ml (both from KC Biological) and $10 \%$ fetal calf serum]. Parasites were subsequently cultivated for up to 2 months in the same medium in the absence of mouse cells. The culture medium was replaced every 4 th day of cultivation. Purified protoscolices were cultivated either as described above (with the initial inclusion of the mouse embryonic cell monolayer) or in Medium 199 at pH 8.0 (for 14 days only) in the absence of mouse cells according to the technique described for E. granulosus protoscolices by Rodriguez-Caabeiro and Casado (1988). Primary vesicular cysts were cultivated as described for oncospheres with the initial inclusion of the mouse embryonic cell monolayer. The whole process was visually monitored in a Leitz Labovert FS microscope.

\section{Experimental hosts and infections}

Selection of C57 BL6/J mice was based on the developmental characteristics of the parasite in this recipient host strain, resembling most closely the morphology of metacestodes in human patients (sparse protoscolex formation in few animals, rapid proliferation of vesicles, embedded in a dense granulomatous area of host origin; Vuitton et al. 1986; Nakao et al. 1990).
Three-month-cold C57BL/6J mice in groups of 3 animals each were injected intraperitoneally (using a syringe and $1.2 \times 40 \mathrm{~mm}$ needles) with the following dose per animal: 3 mice, 50 freshly purified FAub-2 protoscolices; 3 and 2 mice, respectively, 50 protoscolices cultivated in vitro for 14 days in DMEM and Medium 199 , respectively; 3 mice, 50 freshly prepared primary acephalic vesicular cysts; 3 mice, 50 primary acephalic vesicular cysts cultivated in vitro for 14 days; 5 mice (negative control group), an appropriate volume of sterile physiological saline solution.

Another 5 mice were infected by the implantation (Eckert and Pohlenz 1976) of 1 oncospheral cyst per animal, each cyst originating from a single oncosphere cultivated in vitro for a period of 26 days. With regard to the single-cell (egg) origin of these cysts, the individual resultant parasite materials/isolates were considered as clones and are hereafter referred to as clones KF1 to KF5.

For confirmation of the results of infections with FAub-2 protoscolices, 3 mice of another susceptible strain (AKR) were individually infected with 50 freshly purified protoscolices or 50 primary acephalic vesicular cysts of the SLI-144 parasite isolate as described above. At 3 months after inoculation, all animals were euthanised and bled. Sera were stored at $-80^{\circ} \mathrm{C}$. The larval cyst masses were recovered, counted, and measured, and were subsequently stored at $-80^{\circ} \mathrm{C}$.

\section{Immunological and microscopical assays}

Microscopy. The presence of a laminated layer in parasite materials was monitored by light microscopy using either native parasite material or material fixed in $4 \%$ formaldehyde and subsequently stained with PAS.

Direct immunofluorescence. Specific identification of an "Em2-positive" laminated layer was carried out using direct immunofluorescence technique with FITC-labeled mAb G11 according to Deplazes and Gottstein (1991).

Em2-antigen detection by ELISA. Soluble Em2 antigen (released by synthesized laminated layers) was detected in culture-medium supernatants by a double-antibody sandwich ELISA using solidphase and alkaline phosphatase-labeled $\mathrm{mAb}$ G11, the technique having been described in detail elsewhere (Deplazes and Gottstein 1991).

ELISA for mouse serum antibodies. Sera of the mice in each group were pooled. All serum pools were simultaneously tested in parallel runs by ELISA using the following antigens:

1. E. multilocularis vesicular fluid (obtained by aspiration of fluid from primary vesicular cysts of the above-mentioned parasite isolate, which had been cultivated in vitro for 2 months). This antigen represented a crude antigen and contained no murine host components by definition.

2. Immunoaffinity-purified E. multilocularis Em2(G11) antigen (Deplazes and Gottstein 1991).

The ELISA technique employed corresponded to that previously described by Deplazes and Gottstein (1991) except that an alkaline phosphatase-labeled rabbit anti-mouse Ig conjugate (Catalog number 1010-04; Southern Biotechnology Associates, inc., Birmingham) was used.

\section{Results}

\section{In vitro development}

Oncospheres. Post-oncospheral development in vitro involved a rapid loss of oncospheral hooks followed by vesiculation and development of a germinal and Em2positive laminated layer within 14 days of cultivation 

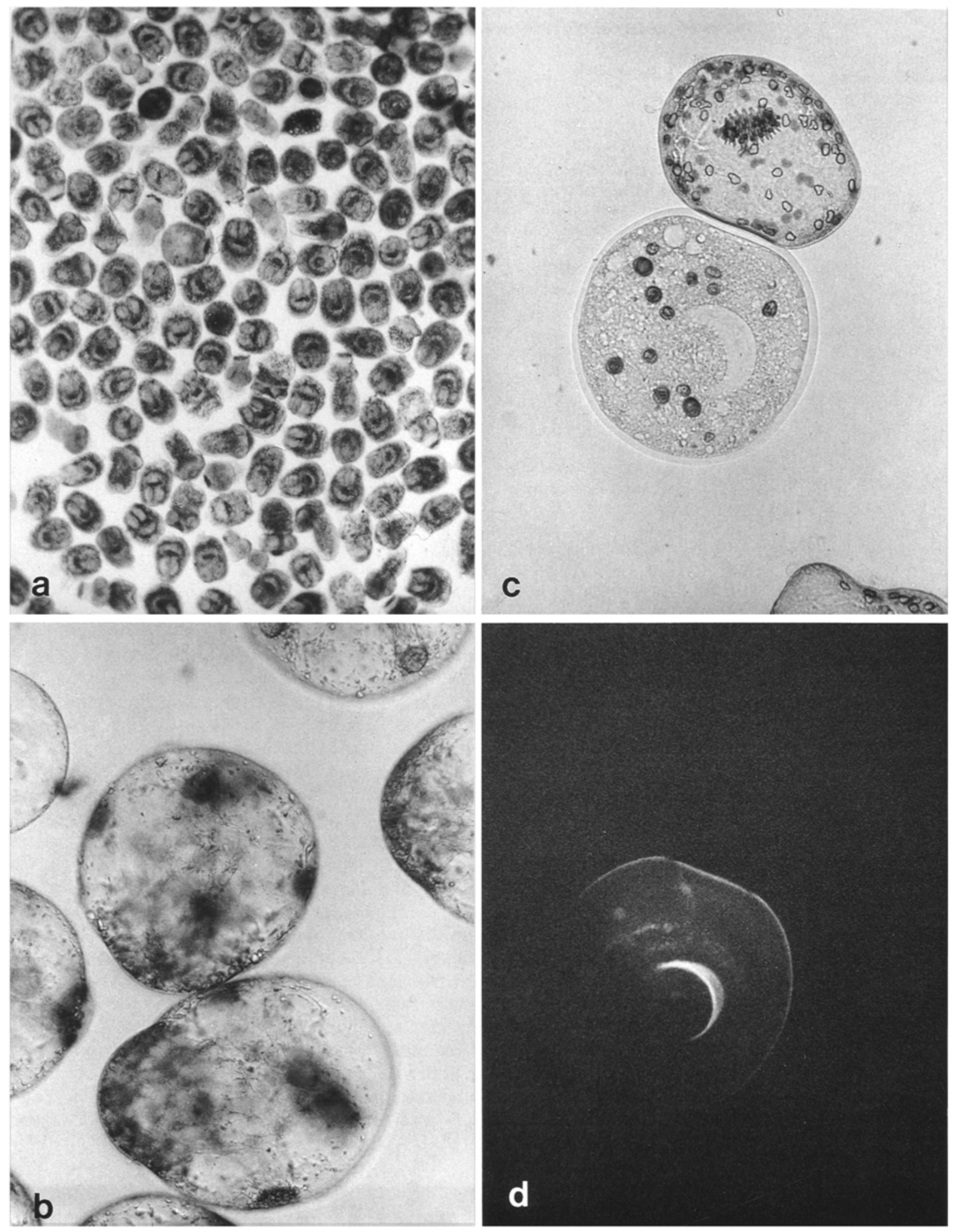

Fig. 1. a Suspension of purified Echinococcus multilocularis protoscolices isolated for subsequent in vitro cultivation and/or infection of experimental rodent hosts $\times 240 ;$ b Vesiculated protoscolices after 14 days of in vitro cultivation $\times 100$; $\mathrm{c}$ Primary acephalic vesicular cysts (note the surrounding laminated layer) and proto-

scolex as viewed under light microscopy $\times 180$; $\mathbf{d}$ The same materials shown in $\mathrm{c}$ but visualized under fluorescence microscopy after immunostaining of the laminated layer with FITC-labeled $\mathrm{mAb}$ G11. Note the lack of surrounding Em2-positive laminated layers for the protoscolices in $\mathbf{c}$ and $\mathbf{d}$ 
Table 1. Direct immunofluorescence analysis of freshly prepared or in vitro cultivated Echinococcus multilocularis oncospheres, protoscolices, and vesicular cysts: detection in culture supernatants of soluble Em 2 antigen from the laminated layer

\begin{tabular}{|c|c|c|c|}
\hline \multirow[t]{2}{*}{$\begin{array}{l}\text { E. multilocularis } \\
\text { larval materials } \\
\text { (FAub-2 isolate) }\end{array}$} & \multicolumn{2}{|c|}{$\begin{array}{l}\text { Reactivity of } \\
\text { larval structures } \\
\text { with FITC-labeled } \\
\text { mAbs }\end{array}$} & \multirow{2}{*}{$\begin{array}{l}\text { Reactivity of } \\
\text { culture supernatant } \\
\text { in the } \mathrm{mAb} \text { G11 } \\
\text { double-antibody } \\
\text { sandwich ELISA }\end{array}$} \\
\hline & $\mathrm{mAb}$ G11 & $\mathrm{mAb} \mathrm{G} 10 / 4^{\mathrm{a}}$ & \\
\hline Oncospheres freshly hatched & - & - & - \\
\hline $\begin{array}{l}\text { Oncospheres cultivated } \\
\text { in vitro for } 14 \text { days }\end{array}$ & + & - & + \\
\hline $\begin{array}{l}\text { Oncospheres cultivated } \\
\text { in vitro for } 2 \text { months }\end{array}$ & + & - & + \\
\hline Fresh protoscolices & - & - & - \\
\hline $\begin{array}{l}\text { Protoscolices cultivated } \\
\text { in vitro for } 14 \text { days, } \\
\text { DMEM }\end{array}$ & - & - & - \\
\hline $\begin{array}{l}\text { Protoscolices cultivated } \\
\text { in vitro for } 14 \text { days, } \\
\text { M199 }\end{array}$ & - & - & - \\
\hline $\begin{array}{l}\text { Protoscolices cultivated } \\
\text { in vitro for } 2 \text { months, } \\
\text { DMEM }\end{array}$ & - & - & - \\
\hline Fresh vesicular cysts & + & - & + \\
\hline $\begin{array}{l}\text { Vesicular cysts cultivated } \\
\text { in vitro for } 14 \text { days }\end{array}$ & + & - & + \\
\hline $\begin{array}{l}\text { Vesicular cysts cultivated } \\
\text { in vitro for } 2 \text { months }\end{array}$ & + & - & + \\
\hline
\end{tabular}

${ }^{a}$ Negative-control $\mathrm{mAb}$ of the same isotype as mAb G11 (photographic documentation in Deplazes and Gottstein 1991). Within 2 months the cyst size varied, ranging up to $6 \mathrm{~mm}$ in diameter; all cysts maintained the Em2and PAS-positive laminated layer.

Protoscolices. The purity of protoscolices obtained for subsequent in vitro cultivation and/or infection is shown in Fig. 1 a. During the 1st week of cultivation, approximately one-third of the protoscolices developed into vesiculated forms, whereas another third showed the development of a posterior bladder; the others remained unchanged (either in- or evaginated; the evaginated forms continued to move around throughout the whole period of in vitro maintenance). Subsequently, refractive asteroidal cells appeared in both developing forms, some of the vesicles growing to a diameter of up to approximately $400 \mu \mathrm{m}$. Native 2-week-old protoscolices cultivated in vitro are shown in Fig. 1 b. No laminated layer became microscopically (native and PAS-stained) or immunomicroscopically (MAb G11-FITC) visible during the 2months course of in vitro cultivation.

Primary vesicular cysts. A small proportion of the cysts cultured in vitro showed a gradual increase in size, reaching diameters of up to several millimeters after 2 months. The majority of the cysts remained unchanged in size or displayed only a small increase in diameter. Initially, the cysts were surrounded by a thick laminated layer (both PAS- and mAb G11-FITC-positive), which grew in relation to the growth of the individual cyst.

\section{Immunological analysis of the parasite material}

Table 1 shows the results of sequential immunofluorescence analysis of fresh or in vitro cultivated Echinococcus multilocularis larval structures as well as the detection of soluble Em2 (laminated layer) antigen in culture-medium supernatant. These findings confirm the microscopical examination of the materials listed above in that oncospheres synthesized an Em2-positive laminated layer within 13 days of in vitro cultivation which was verified by the detection of soluble Em2 antigen in the culture medium supernatant. Vesicular cysts demonstrated the presence of an Em2-positive laminated layer and of soluble $\mathrm{Em} 2$ antigen in the culture-medium supernatant from the beginning of cultivation. Purified protoscolices failed to show any reactivity with mAb G11 before, during, or after in vitro cultivation, confirming their inability to synthesize an Em2-positive laminated layer under the present culture conditions. Examples of simultaneous immunomicroscopical analyses of protoscolices and vesicular cysts are shown in Fig. 1c and Fig. $1 \mathrm{~d}$.

\section{Experimental infections}

To determine whether the different fresh or in vitro cultivated parasite structures were capable of inducing secondary alveolar echinococcosis in vivo, $\mathrm{C} 57 \mathrm{Bl} / 6 \mathrm{~J}$ mice were intraperitoneally infected with the respective materials. Results of the macroscopic examination of necropsied mice are shown in Table 2, demonstrating that only oncospheres and vesicular cysts had the potential to un- 
Table 2. Results of macroscopic and microscopic examination of C57BL/6J mice infected with freshly prepared or in vitro cultivated Echinococcus multilocularis oncospheres, protoscolices, and vesicular cysts

\begin{tabular}{|c|c|c|c|}
\hline \multirow[t]{2}{*}{$\begin{array}{l}\text { E. multilocularis } \\
\text { larval material } \\
\text { used for infection } \\
\text { (FAub-2 isolate) }\end{array}$} & \multirow[t]{2}{*}{$\begin{array}{l}\text { Number of } \\
\text { intraperitoneal } \\
\text { lesions detected } \\
\text { per animal }^{\mathrm{a}}\end{array}$} & \multicolumn{2}{|c|}{$\begin{array}{l}\text { Reactivity of } \\
\text { mouse serum } \\
\text { pools with antigen } \\
\text { in ELISA }\left(\mathrm{A}_{405 \mathrm{~nm}}\right)^{\mathrm{b}}\end{array}$} \\
\hline & & vesicular fluid & $\operatorname{Em} 2(\mathrm{G} 11)$ \\
\hline Fresh protoscolices & $0,0,0$ & 0.181 & 0.034 \\
\hline $\begin{array}{l}\text { Protoscolices cultivated } \\
\text { in vitro for } 14 \text { days, } \\
\text { DMEM }\end{array}$ & $0,0,0$ & 0.307 & 0.041 \\
\hline $\begin{array}{l}\text { Protoscolices cultivated } \\
\text { in vitro for } 14 \text { days, } \\
\text { M } 199\end{array}$ & 0,0 & 0.212 & 0.029 \\
\hline Fresh vesicular cysts & $4,4,6$ & 0.470 & 0.316 \\
\hline $\begin{array}{l}\text { Vesicular cysts cultivated } \\
\text { in vitro for } 14 \text { days }\end{array}$ & $3,6,7$ & 0.570 & 0.277 \\
\hline $\begin{array}{l}\text { Oncospheres cultivated } \\
\text { in vitro for } 26 \text { days }\end{array}$ & $1,1,1,1,1^{\mathrm{c}}$ & 0.334 & 0.291 \\
\hline $\begin{array}{l}\text { Negative controls, } \\
\text { individually tested (mean }+3 \mathrm{SD} \text { ) }\end{array}$ & - & 0.074 & 0.048 \\
\hline
\end{tabular}

a Diameters of lesions varied between 1 and $12 \mathrm{~mm}$

b Positive reactions are shown in boldface, i.e., value for test serum $>$ mean values $+3 \mathrm{SD}$ for negative control sera

${ }^{c}$ Due to the implantation of a single parasite organism, each metacestode recovered was characterized by a large (diameter, $1-3 \mathrm{~cm}$ ) multivesicular, irregularly shaped intraperitoneal lesion attached to and protruding into surrounding organs such as the liver, spleen, and mesenterium dergo further in vivo proliferation by establishing secondary echinococcosis in experimental animals. Serological examination of the infected animal groups (positive/ negative discrimination of the results was based on the mean value +3 SD for negative control mice) revealed that all mice developed a humoral immune response versus a crude $E$. multilocularis (vesicular fluid) antigen, independently of the outcome of parasite survival. Only mice developing secondary alveolar echinococcosis (infections with oncospheres and vesicular cysts) could generate an anti-Em2 antibody response. Experimental infection of AKR mice with protoscolices and vesicular cysts of the SLI-144 produce results identical to those found for the C57BL/6J mice that had been infected with FAub-2 protoscolices; therefore, the corresponding data are not shown in Table 2.

\section{Generation of E. multilocularis clones}

Implantations of single in vitro proliferated oncospheres into individual mice resulted in the generation of five parasite isolates, which by definition of their origin (a single E. multilocularis egg) can be designated as cloned metacestode material, since the introduction of heterologous genomic $E$. multilocularis material could be excluded under the selected experimental conditions.

\section{Discussion}

The development of Echinococcus multilocularis larval or metacestode stages in vitro demonstrated an ability to synthesize an "Em2-positive" laminated layer within a short period of time and under identical cultivation conditions for oncospheres and germinative vesiculated cysts but not for purified protoscolices. It cannot be excluded that other culture conditions or longer culture periods may result in formation of the Em2-positive laminated layer. Consequently, we repeated the cultivation of $E$. multilocularis protoscolices under conditions that had previously been optimized for $E$. granulosus by Rodriguez-Caabeiro and Casado (1988) and had been shown to result in the synthesis of an E. granulosus laminated layer within 9 days of cultivation. Even under these conditions, $E$. multilocularis protoscolices failed to develop a laminated layer.

In previous investigations we had demonstrated that (a) Em2-antigen localization has a morphologically unique restriction to the laminated layer (shown by binding activity to mAb G11) and (b) protoscolices neither displayed binding activity to mAb G11 nor showed any microscopical evidence of the presence of a laminated layer in vivo (Deplazes and Gottstein 1991). In preliminary unpublished experiments, we had observed the inability of protoscolices to synthesize $\mathrm{Em} 2$ in vitro, and these observations were used to design the present study and to establish a working hypothesis that the capability of synthesizing an "Em2-positive" laminated layer is essential for in vivo parasite survival. This is supported by the observation that oncospheres developed the laminated layer within 2 weeks, a period corresponding approximately to that required for a host to generate a specific systemic immune response. In consequence, an immune response directed toward the primary oncosphere surface may become irrelevant in the course 
of infection in that the laminated layer might mask and protect the developing parasite organism. This hypothesis is further supported by the present results, which show that even single oncospheres surrounded by a laminated layer are capable of inducing secondary alveolar echinococcosis as well as vesicular cysts. Host animals subsequently generate an anti-Em2 antibody response; however, this response seems to play no relevant role in controlling parasite survival.

The inability of protoscolices to synthesize "Em2positive" laminated layers was indirectly reflected by the negative Em2 serology of mice infected with protoscolices. It must be emphasized that the inability of protoscolices to synthesize either Em2 antigen or a laminated layer and to develop further in vivo may be applicable only to the present in vitro culture and in vivo infection conditions and to the specific mouse strain investigated (C57BL/6J and AKR). Data suggesting a similar tendency have been provided by Delabre et al. (1987), who used primary $E$. multilocularis cyst inoculation to induce secondary alveolar echinococcosis, and by Al Nahhas et al. (1991), who had to enclose protoscolices in a micropore chamber (preventing access of the parasites to the hots cells) to obtain subsequent protoscolex development.

More generally, our observations contrast with the findings of Yamashita et al. (1962), who injected protoscolices into laboratory rodents and observed the subsequent generation of secondary metacestode proliferation. However, this discrepancy could partly be attributed to differences in the procedures used, as the perfect purification of protoscolices requires extremely careful monitoring due to frequent contamination with vesicular cysts of the same size as protoscolices. In our experiments, such cysts or particles had to be individually removed with sterile glass microcapillaries, despite the new purification procedure described by Thompson et al. (1990). This aspect was not addressed by Yamashita et al. (1962). The administration of 500-2000 protoscolices by the latter authors also appears to be a very high inoculation dose in comparison with the single oncospheres that were used in the present investigation, and Kroeze and Tanner (1986) have shown that the inoculation of as few as three vesicular cysts is sufficient to generate secondary alveolar echinococcosis in C57BL/6J mice. Assuming that a suspension of 500-2000 protoscolices has been contaminated with a few vesicular cysts, subsequent metacestode proliferation could occur without the protoscolices being the source of the proliferating parasite tissue. Our hypothesis may furthermore be supported by the observations of Hinz $(1972 a, b)$, who showed that the rate of parasite growth (after the injection of various parasite preparations) was highly dependent on the cyst number, regardless of the number of protoscolices included.

The ability of our method to generate easily "cloned" E. multilocularis metacestode tissue offers a good means of addressing questions about the genetic homo- or heterogeneity of metacestode material recovered from naturally infected animals (Thompson and Lymbery 1990). As most lahoratories ohtain their $F$ multilomularis isolates by transplanting/injecting parasite material obtained from naturally infected sources (e.g., wild rodents, human patients), the possibility can never be excluded that the source of the parasite lesion might involve several eggs that may even have originated from different adults. Therefore, naturally recovered E. multilocularis metacestodes do not fulfill "clone" requirements.

Future detailed immunological and morphological investigations of these parasite organisms (oncospheres, primary vesicular cysts, and protoscolices) in vivo will help to elucidate the time-dependent cellular events modulating the parasite's biology. Furthermore, inhibition of the potential to synthesize an Em2-positive laminated layer by immunological or chemical means may provide future strategies for the treatment or prevention of infection or disease.

Acknowledgement. We gratefully acknowledge the competent reading of and comments on the manuscript by Prof. J. Eckert.

\section{References}

Ali-Khan Z, Siboo R (1982) Echinococcus multilocularis: immunoglobulin and antibody response in C57BL/6J mice. Exp Parasitol 53:97-104

11 Nahhas S, Gabrion C, Walbaum S, Petavy AF (1991) In vivo cultivation of Echinococcus multilocularis protoscolices in micropore chambers. Int J Parasitol 21:383-386

Jelabre I, Gabrion C, Contat F, Petavy AF, Deblock S (1987) The susceptibility of the Mongolian gerbil (Meriones unguiculatus) and the OF1 mouse strain to Echinococcus multilocularis - ultrastructural aspects of the cysts. Int J Parasitol 17:773-780

Deplazes P, Gottstein B (1990) Genus- and species-specific monoclonal antibodies against Echinococcus multilocularis. Bull Soc Fr Parasitol 8 [Suppl 2]:912

Deplazes P, Gottstein B (1991) A monoclonal antibody against Echinococcus multilocularis Em2 antigen. Parasitology 103:4149

Eckert J, Pohlenz J (1976) Zur Wirkung von Mebendazol auf Metazestoden von Mesocestoides corti und Echinococcus multilocularis. Tropenmed Parasitol 27:247-262

Gottstein B (1985) Purification and characterization of a specific antigen from Echinococcus multilocularis. Parasite Immunol $7: 201-212$

Hinz E (1972a) Die Aufarbeitung des Infektionsmaterials für die intraperitoneale Infektion der Maus mit Echinococcus multilocularis. Tropenmed Parasitol 23:387-390

Hinz E (1972b) Die Entwicklung des sekundären Echinococcus multilocularis in der experimentell infizierten Maus. Eine quantitative Analyse. Tropenmed Parasitol 23:256-265

Kroeze WK, Tanner CE (1986) Echinococcus multilocularis: responses to infection in Mongolian gerbils. Exp Parasitol 61:1-9

Marchiondo AA, Andersen FL (1984) Light microscopy and scanning electron microscopy of the in vitro evagination process of Echinococcus multilocularis protoscolices. Int $\mathrm{J}$ Parasitol 14:151-157

Nakao M, Nakaya K, Kutsumi H 61990) Murine model for hepatic hydatid disease without biohazard. Ipn J Parasitol 39:296-298

Rausch R, Jentoft VL (1957) Studies on the helminth fauna of Alaska. XXXI. Observations on the propagation of larval Echinococcus multilocularis Leuckart, 1863, in vitro. J Parasitol 43:1-8

Rodriguez-Caabeiro F, Casado N (1988) Evidence of in vitro germinal layer development in Echinococcus granulosus cysts. Parasitol Res 74- 55R-56? 
Sakamoto T, Sugimura M (1970) Studies on echinococcosis. XXIII. Electron microscopical observations on histogenesis of larval Echinococcus multilocularis. Jpn J Vet Res 18:131-144

Thompson RCA, Lymbery AJ (1990) Echinococcus: biology and strain variation. Int J Parasitol 20:457-470

Thompson RCA, Deplazes P, Eckert J (1990) Uniform strobilar development of Echinococcus multilocularis in vitro from protoscolex to immature stages. J Parasitol 76:240-247
Vuitton DA, Guerret-Stocker S, Carbillet JP, Mantion G, Miguet JP, Grimaud JA (1986) Collagen immunotyping of the hepatic fibrosis in human alveolar echinococcosis. Z Parasitenkd 72:97-104

Yamashita J, Ohbayashi M, Sakamoto T, Orihara M (1962) Studies on echinococcosis. XIII. Observation on the vesicular development of the scolex of E. multilocularis in vitro. Jpn J Parasitol $10: 85-96$ 